Published in final edited form as:

Contemp Clin Trials. 2018 January ; 64: 35-40. doi:10.1016/j.cct.2017.11.012.

\title{
Study design considerations for the Standardized Treatment Of Pulmonary exacerbations 2 (STOP2): a trial to compare intravenous antibiotic treatment durations in CF
}

\author{
Sonya L. Heltshe, $\mathrm{PhD}^{1,2}$, Natalie E. West, MD, $\mathrm{MHS}^{3}$, Donald R. VanDevanter, $\mathrm{PhD}^{4}$, D. B. \\ Sanders, MD, MS ${ }^{5}$, Valeria V. Beckett, $\mathbf{M S}^{1}$, Patrick A. Flume, MD $^{6}$, and Christopher H. Goss, \\ MD, MS ${ }^{1,2,7}$ on behalf of the STOP Study Group ${ }^{8}$ \\ ${ }^{1}$ CFF Therapeutics Development Network Coordinating Center, Seattle Children's Research \\ Institute, Seattle WA 98105 USA \\ ${ }^{2}$ Department of Pediatrics, University of Washington School of Medicine, Seattle WA 98121 USA \\ ${ }^{3}$ Division of Pulmonary and Critical Care Medicine, Johns Hopkins University School of Medicine, \\ Baltimore MD 21205 USA \\ ${ }^{4}$ Case Western Reserve University School of Medicine, Cleveland OH 44106 USA \\ ${ }^{5}$ Department of Pediatrics, Riley Hospital for Children, School of Medicine, Indiana University, \\ Indiana, IN 46202 USA \\ ${ }^{6}$ Departments of Medicine and Pediatrics, Medical University of South Carolina, Charleston SC \\ 29425 USA \\ ${ }^{7}$ Division of Pulmonary Medicine, Department of Medicine, University of Washington School of \\ Medicine, Seattle WA 98121 USA
}

\section{Abstract \\ BACKGROUND—Pulmonary exacerbations (PEx) in cystic fibrosis (CF) are common and contribute to morbidity and mortality. Duration of IV antibiotic therapy to treat PEx varies widely in the US, and there are few data to guide treatment decisions.}

\begin{abstract}
METHODS-We combined a survey of CF stakeholders with retrospective analyses of a recent observational study of CF PEx to design a multicenter, randomized, prospective study comparing the efficacy and safety of different durations of IV antibiotics for PEx to meet the needs of people with $\mathrm{CF}$ and their caregivers.
\end{abstract}

\footnotetext{
Corresponding author: Sonya L. Heltshe, Seattle Children's Research Institute, M/S CW8-5B, PO BOX 5371, Seattle WA 98145-5005, SONYA.HELTSHE@SEATTLECHILDRENS.ORG, 206-884-1024.

${ }^{8}$ See Data Supplement Appendix A for STOP Study Group

All authors participated in study conception, study design, data interpretation, drafting, revising and editing the manuscript for final approval. SLH, DRV, VVB analyzed the data

This article has a data supplement, which is accessible from this issue's table of contents online at www.atsjournals.org"

Publisher's Disclaimer: This is a PDF file of an unedited manuscript that has been accepted for publication. As a service to our customers we are providing this early version of the manuscript. The manuscript will undergo copyediting, typesetting, and review of the resulting proof before it is published in its final citable form. Please note that during the production process errors may be discovered which could affect the content, and all legal disclaimers that apply to the journal pertain.
} 
RESULTS-IV antibiotic duration was cited as the most important PEx research question by responding CF physicians and top concern among surveyed CF patients/caregivers. During PEx, forced expiratory volume in 1 second ( $\mathrm{FEV}_{1} \%$ predicted) and symptom responses at 7-10 days of IV antibiotics identified two distinct groups: early robust responders (ERR) who subsequently experienced greater FEV ${ }_{1}$ improvements compared to non-ERR (NERR). In addition to greater $\mathrm{FEV}_{1}$ and symptom responses, only $14 \%$ of ERR patients were treated with IV antibiotics for $>15$ days, compared with $45 \%$ of NERR patients.

CONCLUSIONS-A divergent trial design that evaluates subjects' interim improvement in FEV ${ }_{1}$ and symptoms to tailor randomization to IV treatment duration (10 vs. 14 days for ERR, 14 vs. 21 days for NERR) may alleviate physician and patient concerns about excess or inadequate treatment. Such a study has the potential to provide evidence necessary to standardize IV antibiotic duration in CF PEx care -a first step to conducting PEx research of other treatment features.

\section{Keywords}

cystic fibrosis; FEV1; symptoms; pulmonary exacerbation

\section{INTRODUCTION}

Pulmonary exacerbations (PEx) in cystic fibrosis (CF) are a major cause of morbidity linked to disease progression [1][2] and diminished survival [3][4]. They are common and recurring [5], typically treated with antibiotics and increased airway clearance [6]. A systematic review of the literature found scant evidence upon which to base treatment recommendations [7]. Analysis of the CF Foundation (CFF) Patient Registry (CFFPR) demonstrates wide variation in treatment parameters [5] making it difficult to determine optimal practice [6]. This is particularly important as analysis of the CFFPR suggested a lack of recovery of lung function to previous baseline [8]. There are many reports of risk factors for PEx outcomes but nearly all are based on either observational data subject to indication bias [9][10][11] [12], or small single center randomized studies with inconclusive findings [13][14][15][16] [17].

Identification of best PEx treatment practices is hindered by multiple logistic barriers, including variability of presenting signs and symptoms [18], diverse physician and patient objectives for treatment [19], and the range of treatment combinations currently utilized [12]. Ideally, PEx treatment practices could be optimized by conducting a series of randomized controlled studies comparing differences in a single parameter (e.g., treatment durations, home vs. hospital treatment). It has been suggested that studying differences in treatment duration may be the 'most logical' parameter for initial PEx treatment studies [20].

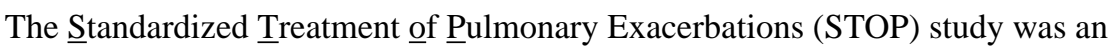
observational pilot study of individuals with $\mathrm{CF}$ who were admitted to the hospital for intravenous (IV) antibiotics for treatment of a PEx. STOP gathered PEx presentation characteristics, physician goals and treatment choices, physician willingness to enroll patients in hypothetical trials, and clinical response[18][19][21], with the ultimate objective of leveraging results to design future controlled interventional trials standardizing aspects of 
CF PEx treatment. While STOP identified a general willingness of CF physicians participating in the study to participate in standardized PEx studies, it was necessary to get broader input from other $\mathrm{CF}$ clinicians, patients and families to understand prioritization of PEx treatment questions and clinical response measures, and specific concerns regarding the design of randomized prospective studies in PEx.

We describe the survey results and report retrospective analyses of the STOP study to rationalize and design a multicenter, randomized, prospective study comparing the clinical efficacy and safety of different durations of IV antibiotic treatment.

\section{MATERIALS AND METHODS}

\section{Stakeholder Surveys}

Two surveys were developed to gauge PEx experiences, perceptions, and research importance among 1) CF patients/caregivers, and 2) CF physicians/providers [Appendices $\mathrm{B}, \mathrm{C}$ in the data supplement]. The patient/caregiver questionnaire was distributed via email to 150 patients and caregivers in the CFF-organized Adult and Patient Family Advisory group (AFA) and conducted via secure, anonymous, electronic data capture using online REDCap database services [22] hosted at the University of Washington. Similarly, a link to the REDCap physician survey was emailed to all CFF Care Center Program Directors (81 adult and 88 pediatric programs) for secure, anonymous completion.

\section{STOP Study}

STOP (clinicaltrials.gov NCT02109822) was an observational pilot study conducted at eleven adult and pediatric CFF Therapeutic Development Network sites between 2014 and 2015[18][19][21]. In brief, CF patients 12 years and older admitted to the hospital for a PEx were assessed for spirometry and patient-reported signs and symptoms throughout treatment and to Day 28. Human subjects approval was granted at all sites by their institutional review boards and written informed consent was obtained from all subjects.

\section{Variables and Statistical Methods}

Spirometry was conducted according to ATS standards [23] and forced expiratory volume in 1 second $\left(\mathrm{FEV}_{1}\right)$ is expressed as percent predicted [24]. Absolute changes in $\mathrm{FEV}_{1} \%$ predicted from admission to Day 7-10, end of IV antibiotic treatment, and Day 28 were calculated. The CF Respiratory Symptom Diary (CFRSD) was scored according to the Chronic Respiratory Infection Severity Score (CRISS), where 100 is the most severe, and 0 the least. Changes in CRISS and FEV ${ }_{1} \%$ predicted from admission to Day 7-10, end of IV, and Day 28 were summarized. We examined response defined as 'early robust response' (ERR) if absolute $\mathrm{FEV}_{1}$ and CRISS improvements from admission to Day 7-10 exceeded specific, candidate thresholds. For $\mathrm{FEV}_{1}$, we assessed response ranges from 5\% to $10 \%$ predicted; for the CRISS we used the minimal clinically important response of 11 units [25]. Patients not meeting the thresholds at Day 7-10 were considered non-ERR (NERR). Candidate ERR thresholds were cross-tabulated with IV treatment duration and subsequent response at end of IV and Day 28. Means, standard deviations and 95\% confidence intervals were used to calculate sample sizes and superiority/non-inferiority margins for a future 
study. All analyses were performed using SAS (version 9.4, SAS Institute Inc., Cary, NC, 2013), and R (version 3.2.1, The R Foundation for Statistical Computing, Vienna, Austria, 2015).

\section{RESULTS}

\section{Physician and Patient/caregiver (AFA) Surveys}

102 of 169 CF physicians (60.4\%) responded to the survey in July 2015: 44\% were pediatric providers, $45 \%$ were adult providers, and $11 \%$ providing care to both, with even distribution across US regions. A majority (73\%) of respondents had $>10$ years' experience in CF care and most $(78 \%)$ worked at centers with $>100$ patients. Just over one third $(\mathrm{n}=52)$ of the AFA completed the patient/caregiver survey in June 2015: 49\% were persons with CF and 51\% were parents, spouses, or partners of persons with CF; $37 \%$ of the surveyed CF population was $<18$ years of age. Nearly all (92\%) reported IV antibiotic treatment of PEx for the person with $\mathrm{CF}$ at some time in the past. Detailed responses to questions regarding current PEx practices, interest in future studies, and clinical endpoints are in the online data supplement (Tables E1, E2). Key findings include: (1) both groups expressed high interest in studies of management of PEx (Table 1); (2) clinicians reported (80\%) and patients/ caregivers assumed (85\%) that antibiotics are selected based on recent culture and susceptibility testing; and (3) there were differences between clinicians and patients/families regarding most important treatment response measures: change in $\mathrm{FEV}_{1}(47 \%$ clinicians vs. $17 \%$ patient/caregivers, respectively) and improvement in symptoms (32\% clinicians vs. $77 \%$ patient/caregivers). Both groups also offered additional comments (Tables E3, E4) with concerns expressed about too short a treatment duration, resulting in incomplete treatment, but also concern for receiving too long of a treatment.

\section{Influence of Survey Results on STOP2 Study Design}

STOP2 is a prospective comparison of different IV antibiotic treatment durations because both clinician and patient/caregiver surveys identified treatment duration as high-priority PEx management question (Table 1). Because the majority of those enrolled in the STOP pilot were adults [18] [19], and because pediatric patients are more likely to be treated with oral or inhaled antibiotics as a first line treatment [26], only STOP data from those 18 years and older were analyzed with the intent to restrict STOP2 to the adult population. The typical range of IV treatment durations across the US is 4-23.5 days [6], but we chose to compare the most common durations from the CFFPR: $10( \pm 1), 14( \pm 1)$, and $21( \pm 3)$ days [27]. To address survey respondents' concerns of potential for overtreatment or undertreatment, we conducted analyses to test a hypothesis that treatment duration inversely correlated with the magnitude of early treatment response, and used different $\mathrm{FEV}_{1}$ thresholds ranging from 5-10\% predicted change from admission to Day 7-10 to evaluate the proportion of patients who might be considered early robust responders (ERR) versus non-ERR (NERR) (Table 2). The Day 7-10 ERR threshold was made more stringent requiring that adults also experience a $\geq 11$ point CRISS improvement from admission (Table 2), the minimal clinically important symptom response in CF [25]. 
Changes in mean $\mathrm{FEV}_{1}$ from admission to Day 7-10 were significantly higher in ERR (range: $14-18 \%$ predicted) than NERR (range: $2-4 \%$ predicted) $(\mathrm{p}<0.05)$, regardless of FEV $_{1}$ threshold studied. Though attenuated, differences between ERR and NERR remained at Day 28 (Figure 1); ERR patients had a mean response at follow-up that was less than their mean response at Day 7-10, while NERR patients had an average $\mathrm{FEV}_{1}$ at follow-up that was greater than their Day 7-10 response (Figure 1).

To study how STOP adults' experience compared to the proposed STOP2 treatment durations of $10( \pm 1), 14( \pm 1)$, and $21( \pm 3)$ days, we categorized STOP treatment durations as abbreviated ( $<12$ days), intermediate (12-15 days), and extended ( $>15$ days). Among 89 STOP adults with complete Day $7-10 \mathrm{FEV}_{1}$ and CRISS, and antibiotic treatment duration information, 18 (20.2\%) received abbreviated, 43 (48.3\%) received intermediate, and 28 (31.5\%) received extended durations. Distributions of antibiotic durations differed substantially by ERR/NERR categorization (Figure 2). Across ERR FEV 1 thresholds ranging from 5\% to $10 \%$ predicted, the proportion of ERR patients receiving extended antibiotic treatments (which ranged from $7.4 \%$ to $12.2 \%$ ) was substantially lower than the corresponding proportion of NERR patients ( $40.0 \%$ to $47.9 \%$ ). Conversely, the proportion of NERR patients receiving abbreviated treatments $(<15 \%$ at all thresholds) was lower than the proportion of ERR patients receiving abbreviated treatments (25-35\% depending on $\mathrm{FEV}_{1}$ threshold). These findings are consistent with a hypothesis that initial clinical response influences length of IV antibiotic treatment. Mean FEV ${ }_{1}$ change from admission to Day 28 for STOP adults stratified by antibiotic treatment duration and ERR/NERR threshold at Day 7-10 are in Data Supplement Table E5.

To design STOP2 to mimic current treatment duration allocation, we compared the proportion of STOP adults receiving abbreviated and extended antibiotic treatments to the proportions of patients that would receive abbreviated and extended treatments were we to apply different thresholds to randomize ERR patients 1:1 to $10( \pm 1)$ days (abbreviated) or $14( \pm 1)$ days (intermediate) and NERR patients 1:1 to receive 14 (intermediate) or $21( \pm 3)$ days (extended) of IV antibiotics (Figure E1). An $\mathrm{FEV}_{1}$ threshold of $7 \%$ predicted would assign $19.7 \%$ to receive abbreviated treatments -similar to the $20.2 \%$ in STOP who did receive $<12$ days treatment. Using an $8 \%$ predicted threshold would assign $32.0 \%$ of patients to receive extended treatments ( $31.5 \%$ in STOP received $>15$ days IV treatment).

Conservatively, undertreating participants with a duration shorter than their providers would have chosen for them was considered higher risk than classifying a higher proportion of participants into NERR, therefore, $8 \%$ change in $\mathrm{FEV}_{1}$ and CRISS improvement of 11 or more at Day 7-10 was chosen to define ERR in STOP2. Figure 3 shows significantly higher $\mathrm{FEV}_{1}$ change in STOP ERR adults by this categorization (12.3\% versus $4.0 \%$, mean diff $=8.3 \%, 95 \% \mathrm{CI}=(4.8 \%, 11.8 \%), \mathrm{p}<0.001)$. An $8 \% \mathrm{FEV}_{1}$ increase and 11 point CRISS reduction was not disproportionately experienced by patients with mild disease: $58 \%$ of ERR and $66 \%$ of NERR had low lung function ( $<50 \%$ predicted) at the start of treatment, and Data Supplement Table E6 shows clinical response in STOP by the chosen threshold and lung function at admission. 


\section{STOP-2 Design and Hypotheses}

Based on the clear divergence in treatment durations and outcome by early response, we suggest two separate hypotheses to test in STOP2: 1) abbreviated (10 \pm 1 day) IV treatment would not be inferior to $14 \pm 1$ day treatment in ERR patients, and 2) extended ( $21 \pm 3$ day) IV treatment would be superior to $14 \pm 1$ day treatment in NERR patients. The proposed STOP2 study schema is shown in Figure 4.

In STOP2, adults with CF who are started on IV antibiotics for a PEx will be recruited to enroll in a randomized, controlled, open-label study designed to evaluate the efficacy and safety of differing durations of treatment. Treatment for the PEx can occur at home or in the hospital and physicians will be provided antibiotic selection and dosing guidelines to minimize variability of care. Participants will be evaluated at day 7-10 of treatment (Visit 2; Figure 4) and based on their categorization into ERR or NERR, patients will be randomized 1:1 to 10 vs. 14 days (ERR) or 14 vs. 21 days (NERR) IV antibiotic treatment, respectively. The primary efficacy endpoint for both ERR and NERR is be absolute change in FEV $1 \%$ predicted from start of treatment to follow-up visit 14 days after scheduled completion of the assigned IV antibiotic treatment (Visit 3)[21]. Secondary and safety endpoints include change in CRISS symptom scores and weight, need for PEx re-treatment within 30 days of finishing IV treatment, time to next PEx (ascertained via the CFFPR), and adverse events. Airway clearance and continuation of chronic medications are encouraged [6]; corticosteroids and changes to antibiotics are permitted prior to randomization.

Randomization will be stratified by location of treatment (exclusively home vs. any hospital days), $\mathrm{FEV}_{1}$ at treatment start ( $<\mathrm{vs} \geq 50 \%$ predicted), history of IV antibiotics in the prior year(0-1 vs $2+)$, and systemic corticosteroid use. Blood and sputum will be collected at each visit for analysis of $\mathrm{C}$-reactive protein, and sputum microbiome.

For ERR patients, we hypothesize that 10 days IV antibiotic treatment (ERR-10) is as safe as and not clinically inferior (in terms of lung function) to 14 days (ERR-14). With 155 subjects per arm, the ERR study has $93 \%$ power $(\mathrm{SD}=9 \%)$ to detect a $3.5 \%$ non-inferiority margin, which preserves $72 \%$ of the treatment effect or $63 \%$ of the lower bound of treatment effect observed in STOP (ERR mean change 12.3\% predicted at Day 28, 95\% CI [9.3, $15.3])$.

The benefit of a prolonged course of IV antibiotics must outweigh potential risks of toxicity, treatment burden, and increased resource utilization. We hypothesize that 21 days (NERR-21) is clinically superior (in terms of lung function) and safe, compared to 14 days (NERR-14) IV antibiotic treatment. A superiority design among NERR requires 285 subjects per arm to have $91 \%$ power $(\mathrm{SD}=9 \%)$ to detect a $2.5 \%$ greater increase in $\mathrm{FEV}_{1}$. Further details on sample size and design treatment effect for both ERR and NERR are in the online Data Supplemental as well as power for secondary endpoints.

We anticipate 1:2 distribution of subjects in the ERR and NERR groups based on our findings in STOP, and assuming 15\% drop out both before randomization (Visit 2) and after, we expect to enroll approximately 1,200 adult CF patients and randomize approximately 1,000 for 880 evaluable at Visit 3. Because STOP2 will enroll a slightly different patient population (including home IV, excluding patients who receive $<7$ days of IV antibiotics), an 
early interim assessment of enrollment and feasibility will be performed, and safety will be overseen throughout the study by an independent data monitoring committee.

\section{DISCUSSION}

We have designed a large scale, randomized, controlled study of IV antibiotic duration for the treatment of PEx that balances concerns of inadequate treatment with the need to establish benefit from prolonged care. Optimizing the duration of antibiotic treatment has the potential for high impact because it is a known source of variability between and within centers [27]. We have incorporated the perceptions of both CF clinicians and patients/ families in design of the study; both groups deem the study of IV antibiotic duration important.

Shorter treatment duration may result in inadequate recovery or early relapse, while longer treatment might be associated with diminishing $\mathrm{FEV}_{1}$ improvement, extra cost and toxicity [27][28][29]. Identifying the optimal antibiotic duration in CF is important because it has implications on treatment decisions, antimicrobial resistance, complications from therapy, health care utilization, missed days of work and/or school, and cost effectiveness [30]. Previous studies in other respiratory infections (e.g. ventilator assisted pneumonia, community acquired pneumonia) have successfully reduced antibiotic burden in the hospital [31][32][33], and we believe the same can be shown for CF PEx. For the CF research community, a standardized duration will allow for the systematic evaluation of other adjunct therapies without the confounding effect of antibiotic duration. This will facilitate controlled trials to optimize other facets of PEx care: location (home versus hospital), administration of mucolytics or steroids, airway clearance techniques, antibiotic selection, route, or combination, etc., all of which are identified gaps in PEx treatment knowledge [6]. A widely accepted antibiotic treatment length has the potential to entice new investigational therapies in this area; the lack of standardized treatment protocols in CF PEx has discouraged drug development specifically targeted at treating acute respiratory events.

Survey response was not complete, which can introduce possible bias or lack of generalizability; however we identified concern expressed by CF clinicians, patients, and caregivers that early assignment to a specific treatment duration might be unacceptable for fear of premature cessation in the absence of improvement, as well as reluctance to commit to prolonged treatment when there is a rapid response. Our analyses of STOP data suggest that dividing a study population into ERR and NERR groups based upon clinical response observed between days 7 and 10 of treatment will likely mitigate those concerns. Patients identified as ERR were much less likely to be treated for extended periods exceeding 15 days and were observed to have minimal additional benefit from intermediate 12-15 day treatments in comparison to abbreviated $<12$ day treatments. Thus, for ERR patients, the important clinical question is whether abbreviated treatment (10 days) provides no worse outcome than treatment for 14 days. In contrast, STOP NERR patients were much more likely to receive extended treatments and appeared to have additional $\mathrm{FEV}_{1}$ improvements during extended treatment, in comparison to intermediate treatment. For these patients, the clinical question is whether extended treatment (18-21 days) provides superior outcomes to treatment for 14 days, and will there be sufficient benefit to outweigh the cost and risks of 
adverse events? While conceivable that patients could respond to the questionnaire at Day $7-$ 10 in a biased manner to influence their ERR/NERR determination, the $8 \% \mathrm{FEV}_{1}$ recovery threshold serves as an impartial measure.

We proposed studying adult patients only because STOP had only $20 \%$ pediatric participation, thus providing insufficient data to adequately estimate treatment response in this population. Although this approach to treatment of PEx (i.e. early assessment of clinical response to determine treatment duration allocation) may be applicable to children, the safety and efficacy of fixed IV antibiotic durations must first be established in CF adults, before tailoring a study to the needs of the pediatric CF patient. It is also important to note that the primary endpoint is change in lung function from start of the PEx, rather than 'baseline' in some period prior to the PEx. We chose this because STOP data showed that approximately $20 \%$ of patients start at their best $\mathrm{FEV}_{1}$ value at the time of IV antibiotic initiation [18] and there are no pre-PEx assessments of symptom scores [21]. Absolute change in $\mathrm{FEV}_{1} \%$ predicted was chosen over relative change for efficiency, though the two endpoints have similar properties [21]. Timing of the final $\mathrm{FEV}_{1}$ measure was chosen to occur 14 days after the end of randomized treatment duration, so not to miss a decline in $\mathrm{FEV}_{1}$ that might occur after the end of IV due to inadequate treatment. However, many patients continue to improve after IV antibiotic treatment [34] including 35\% of STOP participants [21]. Thus, fixing the time interval from antibiotic cessation to measurement of the primary endpoint connotes a common experience for patients.

Research until now has reported varying predictors of PEx non-response or failure to therapy. Therefore, determining the set of appropriate IV antibiotic durations for randomization allocation in a CF population at the time of presentation with a PEx is not possible. There is no reliable presenting phenotype of a patient who would be a good candidate for a shorter IV antibiotic interval (10-14 days) versus an extended duration (1421 days). Half of the participants in STOP2 will receive 14 days of IV treatment (the most common duration currently observed in CF PEx)[27], making this a conservative protocol. We did not consider $<10$ days IV treatment based on a lack of willingness by physicians [19], the patient/caregiver survey results presented here, and reported poorer outcomes in patients treated for $\$$ days [27]. This study design carefully balances the risk of a too-short treatment, with the burden of an extended duration while mitigating the danger that the randomized arms would blur if patients and physicians went off study protocol to address PEx non-response as the participant neared the end of their allocated treatment duration. The 'delayed randomization' also allows physician directed treatment decisions in the 7-10 day period before randomization to tailor patient care without jeopardizing the un-blinded study by potentially introducing confounding therapies.

\section{CONCLUSIONS}

We have designed and implemented the STOP2 study (NCT02781610) to evaluate patient lung function and symptom response after the first week of treatment to then randomize to an antibiotic duration that is appropriate for that patient based on their early response to treatment. The study design was based upon the interests of patients and clinicians and results of STOP data to effectively stratify the STOP-2 study into two patient populations 
with differing PEx treatment needs and unique study hypotheses. We believe this study will provide the foundation for further improvements in PEx management

\section{Supplementary Material}

Refer to Web version on PubMed Central for supplementary material.

\section{Acknowledgments}

We thank Bruce Marshall for his continued support of the STOP program and goal to improve the health of our patients. We thank the Cystic Fibrosis Foundation for the coordinated efforts to collect study data through the CF Foundation Patient Registry (Alex Elbert, Christopher Dowd) and survey responses from the CF Adult and Patient Family Advisory Group (Aliza Fink, Christina Roman, and Kelsey Redkin). Additionally, we would like to thank the patients, families, care providers, and clinic coordinators at CF Centers in STOP and throughout the CFF Therapeutic Development Network for their contributions to the study and the surveys.

Funding: This study was funded by the Cystic Fibrosis Foundation Therapeutics (HELTSH13A1 SANDERS14A0, FLUME13A1, WEST15A0, GOSS13A0) and NIH (P30 DK089507). Dr. Flume's research time was also supported by the South Carolina Clinical Translational Research (SCTR) Institute, with an academic home at the Medical University of South Carolina, through NIH grant number UL1TR000062. There was no role of the sponsor in study design, analysis or interpretation of data, writing of this manuscript, or the decision to submit for publication.

\section{References}

1. Konstan MW, Morgan WJ, Butler SM, Pasta DJ, Craib ML, Silva SJ, Stokes DC, Wohl ME, Wagener JS, Regelmann WE, Johnson CA. Scientific Advisory Group and the Investigators and Coordinators of the Epidemiologic Study of Cystic Fibrosis. Risk factors for rate of decline in forced expiratory volume in one second in children and adolescents with cystic fibrosis. J Pediatr. 2007; 151:134-139. [PubMed: 17643762]

2. Waters V, Stanojevic S, Atenafu EG, Lu A, Yau Y, Tullis E, Ratjen F. Effect of pulmonary exacerbations on long-term lung function decline in cystic fibrosis. Eur Respir J. 2012; 40:61-6. [PubMed: 22135280]

3. de Boer K, Vandemheen KL, Tullis E, Doucette S, Fergusson D, Freitag A, Paterson N, Jackson M, Lougheed MD, Kumar V, Aaron SD. Exacerbation frequency and clinical outcomes in adult patients with cystic fibrosis. Thorax. 2011; 66:680-5. [PubMed: 21680566]

4. Liou TG, Adler FR, Fitzsimmons SC, Cahill BC, Hibbs JR, Marshall BC. Predictive 5-year survivorship model of cystic fibrosis. American journal of epidemiology. 2001; 153:345-52. [PubMed: 11207152]

5. Cystic Fibrosis Foundation Patient Registry: Annual Data Report 2015. Bethesda, MD: Cystic Fibrosis Foundation; 2015.

6. Flume PA, Mogayzel PJ Jr, Robinson KA, Goss CH, Rosenblatt RL, Kuhn RJ, Marshall BC. Clinical Practice Guidelines for Pulmonary Therapies Committee. Cystic fibrosis pulmonary guidelines: treatment of pulmonary exacerbations. Am J Respir Crit Care Med. 2009; 180:802-8. [PubMed: 19729669]

7. Hurley MN, Prayle AP, Flume P. Intravenous antibiotics for pulmonary exacerbations in people with cystic fibrosis. Cochrane Database Syst Rev. 2015; 7:CD009730.

8. Sanders DB, Bittner RC, Rosenfeld M, Hoffman LR, Redding GJ, Goss CH. Failure to recover to baseline pulmonary function after cystic fibrosis pulmonary exacerbation. Am J Respir Crit Care Med. 2010 Sep 1; 182(5):627-32. [PubMed: 20463179]

9. Parkins MD, Rendall JC, Elborn JS. Incidence and risk factors for pulmonary exacerbation treatment failures in patients with cystic fibrosis chronically infected with Pseudomonas aeruginosa. Chest. 2012; 141:485-93. [PubMed: 21835906]

10. Collaco JM, Green DM, Cutting GR, Naughton KM, Mogayzel PJ Jr. Location and duration of treatment of cystic fibrosis respiratory exacerbations do not affect outcomes. Am J Respir Crit Care Med. 2010; 182:1137-43. [PubMed: 20581166] 
11. Sequeiros IM, Jarad N. Factors associated with a shorter time until the next pulmonary exacerbation in adult patients with cystic fibrosis. Chronic respiratory disease. 2012; 9:9-16. [PubMed: 22308550]

12. Heltshe SL, Goss CH, Thompson V, et al. Short-term and long-term response to pulmonary exacerbation treatment in cystic fibrosis. Thorax. 2015

13. Bakker EM, Volpi S, Salonini E, et al. Small airway deposition of dornase alfa during exacerbations in cystic fibrosis; a randomized controlled clinical trial. Pediatr Pulmonol. 2014; 49:154-61. [PubMed: 23913868]

14. Dwyer TJ, Robbins L, Kelly P, Piper AJ, Bell SC, Bye PT. Non-invasive ventilation used as an adjunct to airway clearance treatments improves lung function during an acute exacerbation of cystic fibrosis: a randomised trial. Journal of physiotherapy. 2015; 61:142-7. [PubMed: 26096013]

15. Smith AL, Doershuk C, Goldmann D, Gore E, Hilman B, Marks M, Moss R, Ramsey B, Redding G, Rubio T, Williams-Warren J, Wilmott R, Wilson HD, Yogev R. Comparison of a beta-lactam alone versus beta-lactam and an aminoglycoside for pulmonary exacerbation in cystic fibrosis. $\mathrm{J}$ Pediatr. 1999; 134:413-21. [PubMed: 10190914]

16. Yau YC, Ratjen F, Tullis E, Wilcox P, Freitag A, Chilvers M, Grasemann H, Zlosnik J, Speert D, Corey M, Stanojevic S, Matukas L, Leahy TR, Shih S, Waters V. Randomized controlled trial of biofilm antimicrobial susceptibility testing in cystic fibrosis patients. J Cyst Fibros. 2015; 14:2626. [PubMed: 25453872]

17. Dovey M, Aitken ML, Emerson J, McNamara S, Waltz DA, Gibson RL. Oral corticosteroid therapy in cystic fibrosis patients hospitalized for pulmonary exacerbation: a pilot study. Chest. 2007; 132:1212-8. [PubMed: 17646219]

18. Sanders DB, Solomon GM, Beckett VV, West NE, Daines CL, Heltshe SL, VanDevanter DR, Spahr JE, Gibson RL, Nick JA, Marshall BC, Flume PA, Goss CH. STOP Study Group.

Standardized treatment of pulmonary exacerbations (STOP) study: observations at the initiation of intravenous antibiotics for cystic fibrosis pulmonary exacerbations. J Cyst Fibros. 2017 Apr 28. pii: S1569-1993(17)30101-7. doi: 10.1016/j.jcf.2017.04.005

19. West N, Beckett VV, Jain R, Sanders DB, Nick JA, Heltshe SL, Dasenbrook EC, VanDevanter DR, Solomon GM, Goss CH, Flume PA. STOP Investigators. Standardized treatment of pulmonary exacerbations (STOP) study: physician treatment practices and outcomes for individuals with cystic fibrosis with pulmonary exacerbations. J Cyst Fibros. 2017 Apr 27. pii: S1569-1993(17)30099-1. doi: 10.1016/j.jcf.2017.04.003

20. Goss $\mathrm{CH}$. The next chapter for the treatment of acute pulmonary exacerbation in CF: What is the next study. Pediatr Pulmonol. 2015; 41S:128-129.

21. VanDevanter DR, Heltshe SL, Spahr J, Beckett VV, Daines CL, Dasenbrook EC, Gibson RL, Jain R, Sanders DB, Goss CH, Flume PA. STOP Study Group. Rationalizing endpoints for prospective studies of pulmonary exacerbation treatment response in cystic fibrosis. J Cyst Fibros. 2017 Apr 21. pii: S1569-1993(17)30100-5. doi: 10.1016/j.jcf.2017.04.004

22. Harris PA, Taylor R, Thielke R, Payne J, Gonzalez N, Conde JG. Research electronic data capture (REDCap)--a metadata-driven methodology and workflow process for providing translational research informatics support. Journal of biomedical informatics. 2009; 42:377-81. [PubMed: 18929686]

23. American Thoracic Society. Standardization of Spirometry, 1994 Update. Am J Respir Crit Care Med. 1995; 152:1107-36. [PubMed: 7663792]

24. Quanjer PH, Stanojevic S, Cole TJ, Baur X, Hall GL, Culver BH, Enright PL, Hankinson JL, Ip MS, Zheng J, Stocks J. ERS Global Lung Function Initiative. Multi-ethnic reference values for spirometry for the 3-95-yr age range: the global lung function 2012 equations. Eur Respir J. 2012; 40:1324-43. [PubMed: 22743675]

25. Goss CH, Caldwell E, Gries KS, et al. Validation of a novel patient-reported respiratory symptoms instrument in cystic fibrosis CFRSD-CRISS. Pediatr Pulmonol. 2013; S36:295-96.

26. Wagener JS, Rasouliyan L, VanDevanter DR, Pasta DJ, Regelmann WE, Morgan WJ, Konstan MW. Oral, inhaled, and intravenous antibiotic choice for treating pulmonary exacerbations in cystic fibrosis. Pediatr Pulmonol. 2013; 48:666-673. [PubMed: 22888106] 
27. VanDevanter DR, Flume PA, Morris NJ, Konstan MW. Probability of IV antibiotic retreatment within thirty days is associated with duration and location of IV antibiotic treatment for pulmonary exacerbation in cystic fibrosis. J Cyst Fibros. 2016 Nov; 15(6):783-790. [PubMed: 27139161]

28. Bertenshaw C, Watson AR, Lewis S, Smyth A. Survey of acute renal failure in patients with cystic fibrosis in the UK. Thorax. 2007; 62:541-5. [PubMed: 17234661]

29. Prayle A, Smyth AR. Aminoglycoside use in cystic fibrosis: therapeutic strategies and toxicity. Curr Opin Pulm Med. 2010; 16:604-10. [PubMed: 20814306]

30. Wolter JM, Bowler SD, Nolan PJ, McCormack JG. Home intravenous therapy in cystic fibrosis: a prospective randomized trial examining clinical, quality of life and cost aspects. Eur Respir J. 1997; 10:896-900. [PubMed: 9150331]

31. Chastre J, Wolff M, Fagon JY, Chevret S, Thomas F, Wermert D, Clementi E, Gonzalez J, Jusserand D, Asfar P, Perrin D, Fieux F, Aubas S. PneumA Trial Group. Comparison of 8 vs 15 days of antibiotic therapy for ventilator-associated pneumonia in adults: a randomized trial. JAMA. 2003; 290:2588-98. [PubMed: 14625336]

32. Singh N, Rogers P, Atwood CW, Wagener MM, Yu VL. Short-course empiric antibiotic therapy for patients with pulmonary infiltrates in the intensive care unit. A proposed solution for indiscriminate antibiotic prescription. Am J Respir Crit Care Med. 2000; 162:505-11. [PubMed: 10934078]

33. Uranga A, España PP, Bilbao A, et al. Duration of antibiotic treatment in community-acquired pneumonia: a multicenter randomized clinical trial. JAMA Intern Med. 2016; 176:1257-65. [PubMed: 27455166]

34. Waters V, Stanojevic S, Klingel M, Chiang J, Sonneveld N, Kukkar R, Tullis E, Ratjen F. Prolongation of antibiotic treatment for cystic fibrosis pulmonary exacerbations. J Cyst Fibros. 2015; 14:770-6. [PubMed: 26265220] 

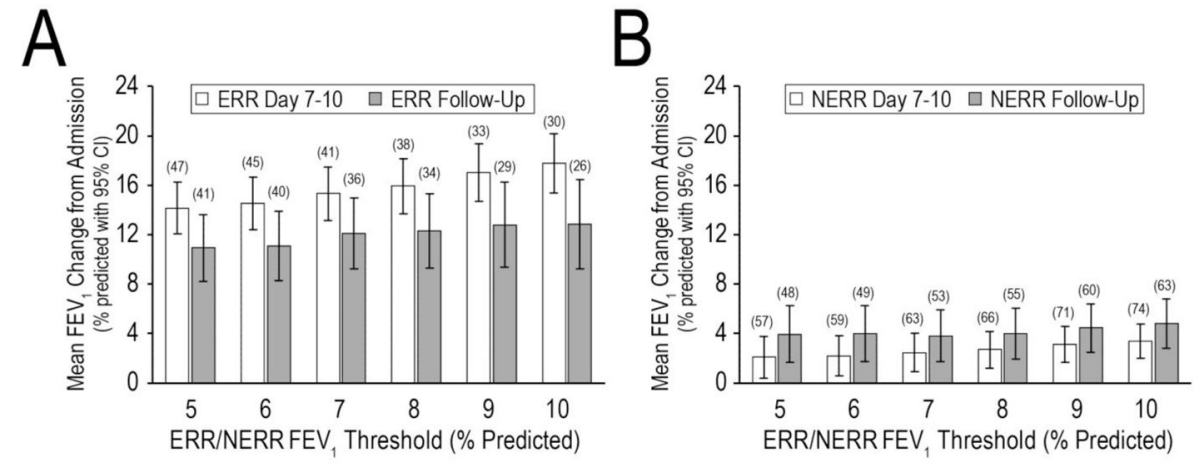

Figure 1. Mean FEV 1 change from admission through Day 7-10 and Follow-Up (Day 28) by ERR/NERR threshold

Panel A, Patients meeting the $\mathrm{FEV}_{1}$ improvement threshold and having a CRISS improvement of $\geq 11$ points at Day 7-10 (ERR). Panel B, patients not meeting both the ERR $\mathrm{FEV}_{1}$ threshold and CRISS criteria at Day 7-10 (NERR). The X-axis shows the Day 7-10 $\mathrm{FEV}_{1}$ change exceeded by ERR patients. Mean $\mathrm{FEV}_{1}$ changes at Day 7-10 are shown in white, mean $\mathrm{FEV}_{1}$ changes at Follow-Up are shown in gray. Sample sizes are shown in parentheses; bars are 95\% confidence intervals for means. Day 7-10 total $n=104$ with both $\mathrm{FEV}_{1}$ and CRISS; Day 28 Follow-Up total $\mathrm{n}=89$ with $\mathrm{FEV}_{1}$. 

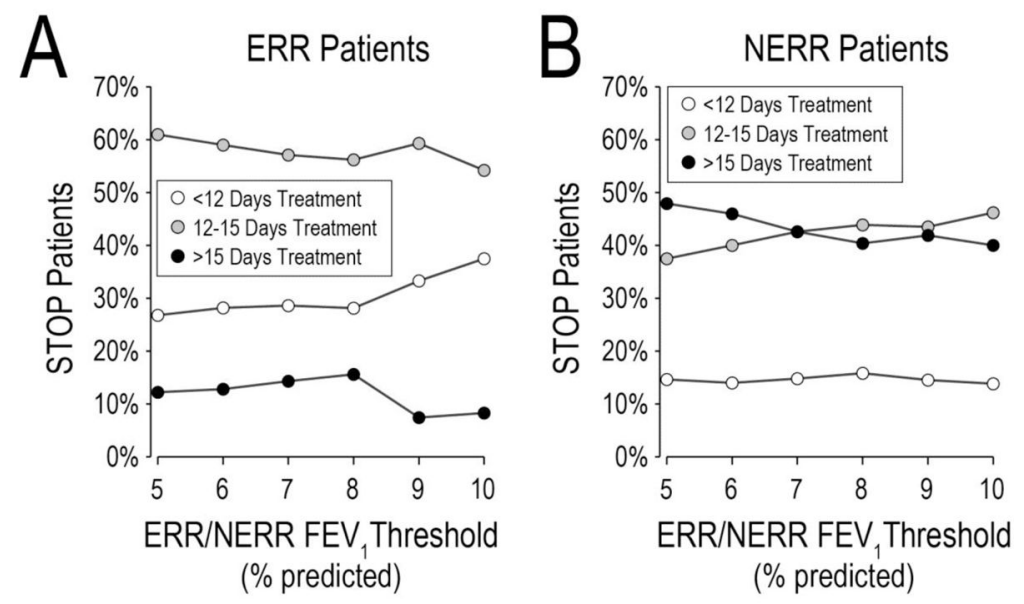

Figure 2. Categorical antibiotic treatment durations for ERR and NERR patients across different ERR FEV 1 thresholds

Panel A, STOP ERR adults; Panel B, STOP NERR adults. Open circles, patients treated less than 12 days. Gray circles, patients treated between 12 and 15 days. Black circles, patients treated $>15$ days. Total $n=89$ with FEV1 and CRISS at Day7-10 and treatment duration recorded. 


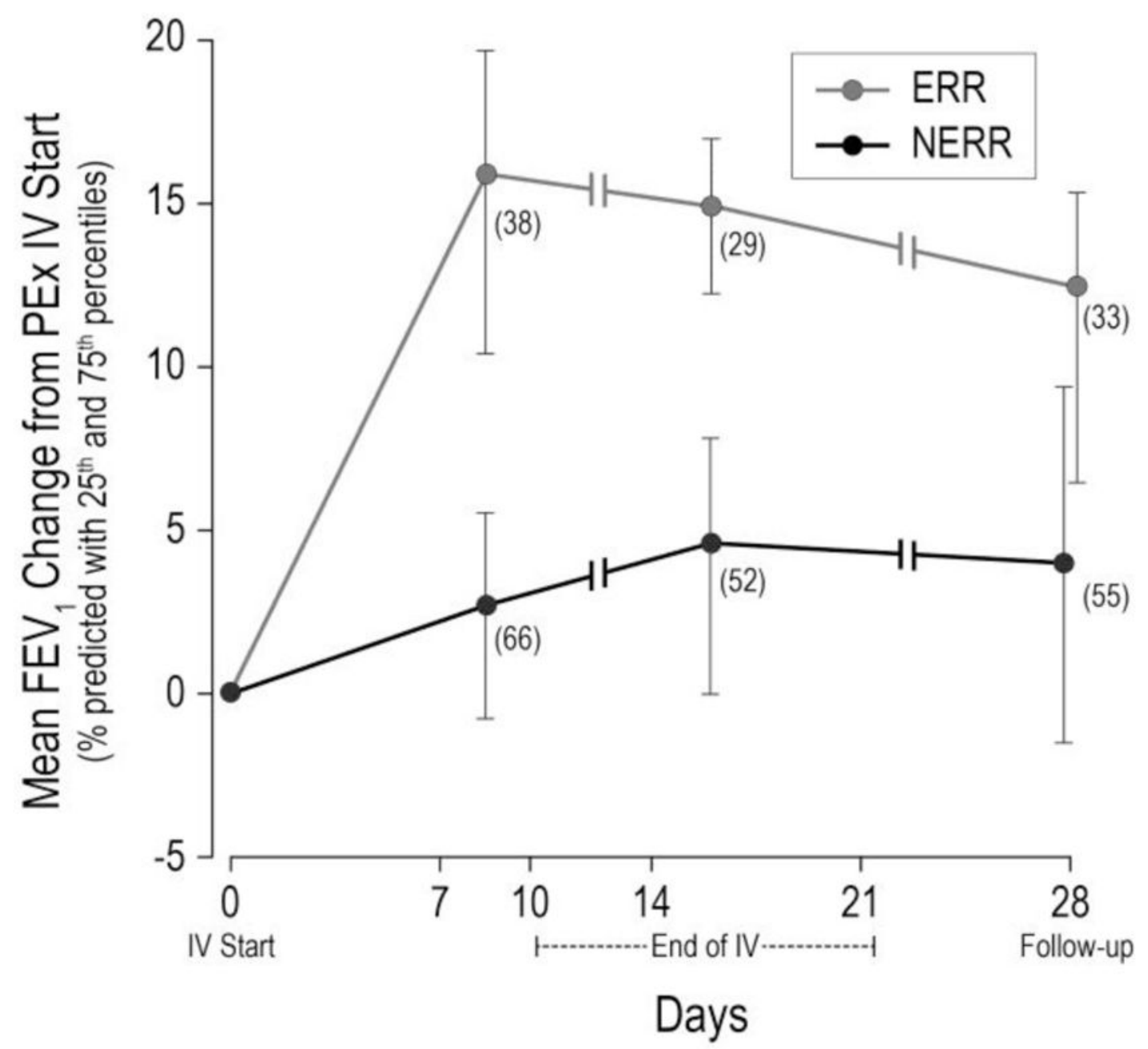

Figure 3. Mean absolute change in $\mathrm{FEV}_{1} \%$ predicted in STOP adults from start of IV antibiotic treatment to Day 7-10, end of IV treatment, and Day 28, by ERR $\left(\mathrm{FEV}_{1} \geq 8 \%\right.$ and CRISS improvement $\left.\geq 11\right)$ and NERR $\left(\mathrm{FEV}_{1}<8 \%\right.$ or CRISS improvement $<11$ ). Vertical lines span from $25^{\text {th }}$ to $75^{\text {th }}$ percentiles; $n$ at each time point shown on figure. 


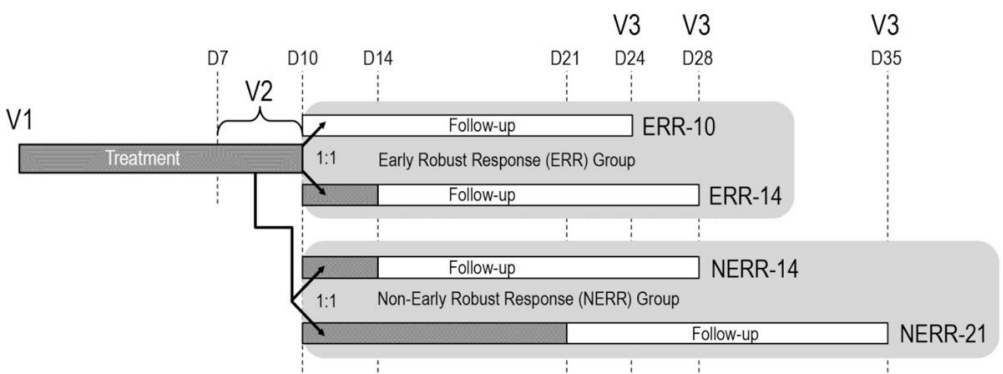

Figure 4. STOP2 Study Schema

Patients enrolled in the study begin receiving IV antibiotics at Visit 1. Their change in FEV 1 and CRISS from Visit 1 is evaluated at Visit 2, between 7 and 10 days after Visit 1. Patients with an $\mathrm{FEV}_{1}$ improvement of $\geq 8 \%$ predicted and CRISS improvement of $\geq 11$ points are allocated to the ERR (Early Robust Rersponse) study branch, where they will be randomized 1:1 to receive either $10( \pm 1)$ or $14( \pm)$ total days of antibiotic treatment. All other patients are allocated to the non-ERR (NERR) study branch, where they will be randomized 1:1 to receive either $14( \pm 1)$ or $21( \pm 3)$ total days of antibiotic treatment. Dark gray bars, IV antibiotic treatment; white bars, post-treatment follow-up; V, Study Visit; D, Study Day. 


\section{Table 1}

Ranking of clinical trial questions for improving treatment of pulmonary exacerbations

\begin{tabular}{llclc} 
Rank & Clinician Responses & Higher Priority $\boldsymbol{a}$ & Patient/Family Responses & Higher Priority $\boldsymbol{a}$ \\
\hline 1 & Antibiotic treatment duration & $73 \%$ & Site of treatment (home, hospital) & $51 \%$ \\
2 & 1 vs. 2 antibiotics for $P a b$ & When to start antibiotics & $51 \%$ \\
3 & Continuous infusion of $\beta$-lactam & $38 \%$ & Antibiotic route(s) & $43 \%$ \\
4 & Site of treatment (home, hospital) & $35 \%$ & Antibiotic treatment duration & $40 \%$ \\
5 & Use of corticosteroids & $32 \%$ & Use of corticosteroids & $20 \%$ \\
\hline
\end{tabular}

${ }^{a}$ Proportions of respondents identifying topic as $1^{\text {st }}$ or $2^{\text {nd }}$ highest priority to study

${ }^{b}$ Pseudomonas aeruginosa airway infection 

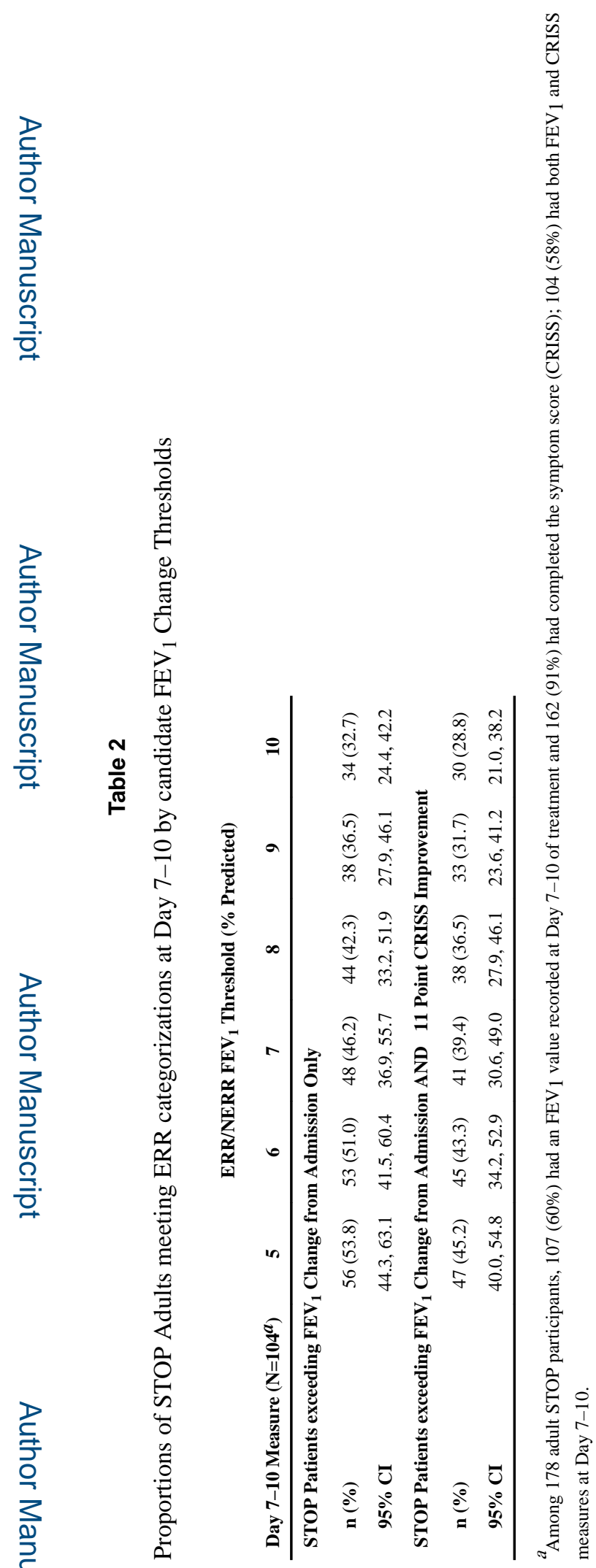

Contemp Clin Trials. Author manuscript; available in PMC 2019 January 01. 